\title{
Implementing and reasoning about hash-consed data structures in Coq
}

\author{
Thomas Braibant • Jacques-Henri Jourdan • \\ David Monniaux
}

November 2013

\begin{abstract}
We report on four different approaches to implementing hash-consing in Coq programs. The use cases include execution inside Coq, or execution of the extracted OCaml code. We explore the different trade-offs between faithful use of pristine extracted code, and code that is fine-tuned to make use of OCaml programming constructs not available in Coq. We discuss the possible consequences in terms of performances and guarantees. We use the running example of binary decision diagrams and then demonstrate the generality of our solutions by applying them to other examples of hash-consed data structures.
\end{abstract}

\section{Introduction}

Hash-consing is a programming technique used to share identical immutable values in memory, keeping a single copy of semantically equivalent objects. It is especially useful in order to get a compact representation of abstract syntax trees. A hashconsing library maintains a global pool of expressions and never recreates an expression equal to one already in memory, instead reusing the one already present. In typical implementations, this pool is a global hash-table. Hence, the phrase hash-consing denotes the technique of replacing node construction by a lookup in a hash table returning a preexisting object, or creation of the object followed by insertion into the table if previously nonexistent. This makes it possible to get maximal sharing between objects, if hash-consing is used systematically when creating objects.

The research leading to these results has received funding from the Agence nationale de la recherche (ANR) under project ' VERASCO' and the European Research Council under the European Union's Seventh Framework Programme (FP/2007-2013) / ERC Grant Agreement nr. 306595 'STATOR'

T. Braibant · J.-H. Jourdan

Inria Paris-Rocquencourt, Domaine de Voluceau, BP 105, 78153 Le Chesnay, France

E-mail: thomas.braibant@inria.fr, jacques-henri.jourdan@inria.fr

D. Monniaux

CNRS / VERIMAG Centre Équation, 2, avenue de Vignate, 38610 Gières, France

E-mail: david.monniaux@imag.fr 
Moreover, a unique identifier is given to each object, allowing fast hashing and comparisons. This makes it possible to do efficient memoization (also known as dynamic programming): the results of an operation are tabulated so as to be returned immediately when an identical sub-problem is encountered.

Hash-consing and memoization are examples of imperative techniques that are of prime importance for performance. The typical way of implementing hashconsing (a global hash table) does not translate easily into Coq. The reason is that the Gallina programming language at the heart of the Coq proof assistant is a purely applicative language, without imperative traits such as hash tables, pointers, or pointer equality. In the following, we discuss how hash-consing can be implemented using the Coq proof assistant (without modifying it) with two possible use cases in mind:

- execution inside Coq with reasonable efficiency, e.g. for proofs by reflection;

- efficient execution when extracted to OCaml, e.g. for use in a model-checking or static analysis tool proved correct in Coq.

More precisely, we present a few design patterns to implement hash-consing and memoization on various examples, and we use binary decision diagrams (BDDs) as our prime example. We evaluate these design patterns based on several criterion. For instance, we discuss for each pattern how we handle the maximal sharing property and how easy it is to prove the soundness of our implementation. We also discuss whether or not it is possible to compute inside Coq with a given solution.

Reading this article. In the following, we will try to separate some implementation details or Coq specific comments from the main text of the article. These remarks, titled "For the practical mind", can be safely skipped on the first reading.

Outline. This paper is organized as follows. Since hash-consing can be seen as a particular kind of memoization, where one memoizes constructor applications, we begin this paper with a short review of the techniques that are available in Coq in order to memoize functions $(\S 2$. Then, we give a short primer on BDDs in $\S 3$ and present the "menu" of our four implementations of BDD libraries in $\S 4$. These implementations are subsequently described in $\S 5$ and $\S 6$ and use different design patterns. We discuss the pros and cons of each design pattern in $\S 7$. We adapt these design patterns to implement strong reduction of hash-consed lambda-terms in $\S 8$. We discuss related work and conclude this paper in $\S 9$.

\section{Memoization in Coq: state of the art}

Generally speaking, there are two ways to use Coq code: either to execute it inside Coq, e.g., to define reflective decision procedures; or to extract it to OCaml (or other supported languages), then compile the extracted code. These two usages impose constraints on the possible design patterns. In the remainder of this section, we describe what the state of the art in memoization approaches is with respect to these two use cases. 


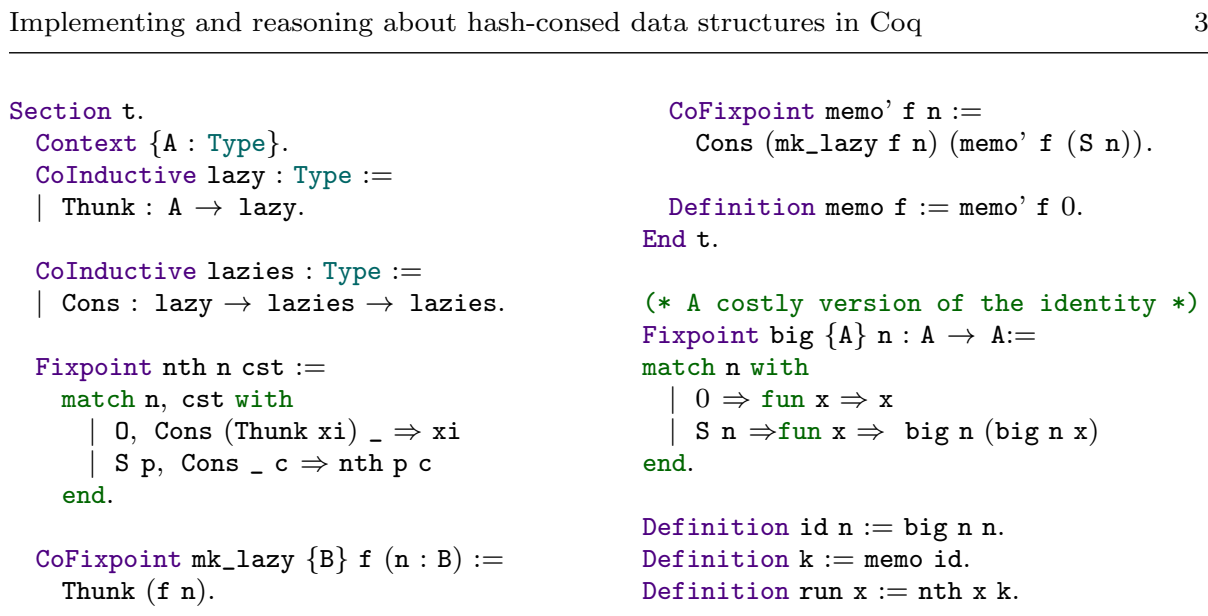

Fig. 1: Memoization using co-inductives

\subsection{State monad}

The first idea to implement memoization in Coq is to use a dedicated data structure to tabulate function calls: that is, use a finite map of some sort to store the pairs (argument, result) for a given function. Users of a library implemented in this fashion must, then, thread this state through the program using a state monad.

While this solution always works, it has non-negligible verification cost: we must prove that the bindings in the memoization table are correct, i.e., the values held in the table correspond to the result of the would-be corresponding function calls.

Then, if we memoize a function of type $A \rightarrow B$, we get a function of type $\mathrm{A} \rightarrow \mathrm{M} \mathrm{B}$, where $\mathrm{M}$ is the type constructor associated with our state monad. Even if the latter seems equivalent to the former, these types are different: therefore, this tiny modification (memoizing a function) requires modifying every caller of the memoized function to use a monadic style.

Since it is cumbersome to perform all computations inside a state monad, we continue this section with a review of other (partial) solutions to the memoization problem.

\subsection{Shallow memoization}

It is possible in some cases to make a shallow embedding of this memoization table in Coq. Melquiond 17 describes how to use co-inductive objects as a cache that stores previously computed values of a given function. In this section, we present his clever idea in Fig. 1. with a few cosmetic changes ${ }^{1}$. A lazy value is defined as a co-inductive, which will effectively be represented as a thunk when executed by Coq's virtual machine, and will be extracted to OCaml as a Lazy.t thunk. A thunk

\footnotetext{
1 We found out that similar definitions actually made their way into Coq's standard library under the name StreamMemo.v, yet we believe that this idea deserves more explanation than what is available.
} 
is a term whose evaluation is frozen until it is actually needed (lazy evaluation), then the computed value is cached in the thunk so that it is instantly returned in case the term is evaluated again (thus, performing a kind of memoization).

Then, lazies represents a stream (an infinite list) of thunks, that can be peeked at using nth. Each time the virtual machine has to destruct the cst argument of nth, it will remember the two arguments of this cons cell. Then, it suffices to build a stream memo that computes the value of a function $f$ for each possible natural number: in practice, $f$ will be evaluated lazily, and only once per input value.

As an example, the following snippet of code memoizes the time-consuming function id. (Remark that it also demonstrates that the intermediate calls of id are not memoized.)

Time Eval vm_compute in run 26. (* 6s *)

Time Eval vm_compute in run 26. (* 0s *)

Time Eval vm_compute in run 25. (* 3s *)

Time Eval vm_compute in run 25 . (* 0 s $*)$
Time Eval vm_compute in id 25. (* 3s *)

Time Eval vm_compute in id 25. (* 3s *)

Note that this clever trick is not limited to functions over Peano numbers, and could be adapted for branching structures, e.g. memoizing functions over binary numbers. Unfortunately, it seems hard to adapt this idea to memoize recursive calls: consider for instance the function

Fixpoint $\exp \mathrm{n}:=\operatorname{match} \mathrm{n}$ with $0 \Rightarrow 1 \mid \mathrm{S} \mathrm{n} \Rightarrow \exp \mathrm{n}+\exp \mathrm{n}$ end.

In order to compute exp using a linear number of recursive calls we need a memoizing fixed-point combinator rather than a way to memoize a predefined function.

(To be complete, let us add that functions $f:$ nat $\rightarrow$ A that can be expressed as the iteration of a given function $\mathrm{g}: \mathrm{A} \rightarrow \mathrm{A}$, can actually be memoized using the aforementioned technique. While we obviously could rewrite exp to fit into that scheme, this is not the case of the other functions that we use through our developments.)

This problem is somewhat representative of the one that we have to face when it comes to BDDs: first, memoizing fixed-point combinators are crucial in our work; second, the function that we memoize depends on the global state of the hash-consing machinery, which evolves through times. In both cases, we fall out of the scope of the above trick: there is no predefined function to memoize.

\subsection{Memoization in extracted code}

Now, we turn to a partial solution to the memoization problem, that works when the user is solely interested in executing the code extracted from a Coq program.

Vafeiadis 21] introduced recently a restricted form of mutable state called adjustable references. The idea is that adjustable references store some internal value that can only be updated in innocuous ways. That is, adjustable references are equipped with an observation function; and the update function ensures that the result of the observations remain equals through updates. It is therefore possible to adjust the values held in the references in a way that changes, e.g., the costs of some computations, yet does not change the result of these computations. 

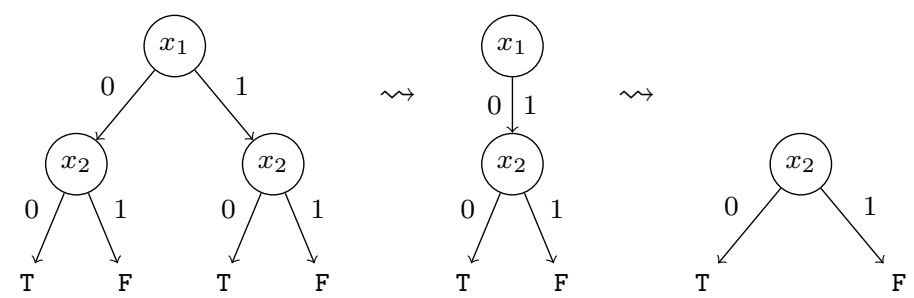

Fig. 2: The BDD for the function $f(0,0)=\mathrm{T}, f(0,1)=\mathrm{F}, f(1,0)=\mathrm{T}, f(1,1)=\mathrm{F}$

Vafeiadis demonstrated how to use adjustable references to memoize a function $f$ by tabulating its results: the adjustable reference internal state is a finite map, while the observation function simply returns $f$. Updating the contents of the finite map does not change the observation function; but subsequent reads may make use of the fact that a value was previously computed and stored inside the memoization cache. We refer the reader to Vafeiadis' article for more details about this idea.

Remark that it is again the case that this technique does not scale to the definition of memoizing fixed-point combinators. Therefore, adjustable references do not quite fit our needs either.

\subsection{Summary}

Out of the three memoization techniques available in Coq that we described, only the first one suits our need: it makes it possible to define memoizing fixed-point combinators. In this paper, we use this state monad approach, then we will come up with a new idiom.

\section{A primer on binary decision diagrams}

In the following, we use "reduced ordered binary decision diagrams" (ROBDDs, BDDs for short) as a running example of hash-consed data structures. BDDs are representations of Boolean functions and are often used in software and hardware formal verification tools, in particular model checkers 16.

The data structure. A Boolean function $f:\{0,1\}^{n} \rightarrow\{0,1\}$ can be represented as a complete binary tree with $2^{n}-1$ decision nodes, labeled by variables $x_{i}$ according to the depth $i$ from the root (thus the adjective ordered); edges are labeled 0 and 1 ; leaves are labeled $\mathrm{T}$ (for true) or $\mathrm{F}$ (for false). The semantics of such a diagram is: $f\left(x_{1}, \ldots, x_{n}\right)$ is obtained by traversing from the root and following the 0 or 1 edge of a node labeled by $x_{i}$ according to the value of $x_{i}$.

Such a tree can be reduced by merging identical subtrees, thus becoming a connected directed acyclic graph (DAG; see second diagram in Fig. 2); furthermore, decision nodes with identical children are removed (see third diagram in Fig. 2). These transformations preserve semantics. The reduced representation is canonical: given a variable ordering $x_{1}, \ldots, x_{n}$, a function is represented by a unique ROBDD. 
Building BDDs. In practice, one directly constructs the reduced tree; let us see how. All BDD nodes allocated so far are stored in a global hash table, so that a new object is created only if not already in the hash table: there are never two identical objects at two different locations in memory, a crucial invariant that must be maintained throughout the execution.

Each BDD node is given a unique identifier: for instance, the current value of a global 64-bit counter incremented following each allocation ${ }^{2}$ Since a new object is never created if an identical object already exists, two objects are equal if and only if they have the same unique identifier. The equality test, which is usually expensive over tree structures, since it requires full traversal, is instead implemented by a very fast comparison of unique identifiers.

Unique identifiers are also used for hashing. When attempting to construct a node $\left(v, b_{0}, b_{1}\right)$ where $v$ is the variable, $b_{0}$ is the subtree labeled 0 and $b_{1}$ is the subtree labeled 1 , one computes the hash value of the node as $H\left(v, u_{0}, u_{1}\right)$ where $u_{0}$ and $u_{1}$ are the respective unique identifiers of the subtrees $b_{0}$ and $b_{1}$. This hash value is then used to look up the node in the hash table. Again, such shallow hashing is considerably faster than having to traverse the tree structures.

Operations on BDDs. The principal way to build BDDs is to combine the diagrams of two functions $f$ and $g$ in order to obtain the BDD for other functions such as $f \wedge g, f \vee g$ of $f \oplus g$. The basic combinator of BDDs is called node melding [16]. Intuitively, melding corresponds to a traversal of the internal nodes of two BDDs to build a third diagram. This traversal respects the order on variables, and thus, the third diagram will naturally be ordered. Then, all binary operations on BDDs are defined as one particular instance of melding.

Suppose that we have two nodes $\alpha=(v, l, h)$ and $\alpha^{\prime}=\left(v^{\prime}, l^{\prime}, h^{\prime}\right)$. The melding of $\alpha$ and $\alpha^{\prime}$, denoted by $\alpha \diamond \alpha^{\prime}$ is defined as follows:

$$
\alpha \diamond \alpha^{\prime}= \begin{cases}\left(v, l \diamond l^{\prime}, h \diamond h^{\prime}\right) & \text { if } v=v^{\prime} \\ \left(v, l \diamond \alpha^{\prime}, h \diamond \alpha^{\prime}\right) & \text { if } v<v^{\prime} \\ \left(v^{\prime}, \alpha \diamond l^{\prime}, \alpha \diamond h^{\prime}\right) & \text { if } v>v^{\prime}\end{cases}
$$

Then, the binary operation $\diamond$ is entirely defined by the results of

$$
\mathrm{F} \diamond \alpha \quad \alpha \diamond \mathrm{F} \quad \mathrm{T} \diamond \alpha \quad \alpha \diamond \mathrm{T}
$$

For instance, the conjunction operation $f \wedge g$ can be defined by melding the BDDs for $f$ and $g$, and using the following rewrite rules for the leaf cases:

$$
\mathrm{F} \diamond \alpha \rightarrow \mathrm{F} \quad \alpha \diamond \mathrm{F} \rightarrow \mathrm{F} \quad \mathrm{T} \diamond \alpha \rightarrow \alpha \quad \alpha \diamond \mathrm{T} \rightarrow \alpha
$$

For the sake of clarity, we focus on binary operations in the following: they are representative of the difficulties we had to face 3 .

\footnotetext{
2 In certain implementations, the unique identifier is the address of the node; this supposes that objects never change address, which is not the case in OCaml.

3 Note that we only need two rewrite rules for commutative binary operations.
} 
Memoization. The fact that each node has a unique identifier also makes it possible to memoize the results of BDD operations. One keeps a map from sub-problems (a pair of nodes $\alpha$ and $\alpha^{\prime}$ ) to nodes (the result of $\alpha \diamond \alpha^{\prime}$ ), so as to be returned immediately when a previously solved sub-problem is encountered.

In a BDD library, memoization is crucial to implement the or/and/xor operations with time complexity in $O(|a| \cdot|b|)$ where $|a|$ and $|b|$ are the sizes of the inputs: as the function is executed, its results on the subtrees of the original problem are stored in a structure indexed by $\left(u_{a}, u_{b}\right)$ where $u_{a}$ and $u_{b}$ are the unique identifiers of the $a$ and $b$ inputs. In contrast, the naive approach has exponential complexity, since the function may be evaluated exponentially often on the same couple of subtrees.

\section{Implementing BDDs in Coq.}

The following two sections $\S 5$ and $\S 6$ describe a total of four implementations of BDDs in Coq. To make things clear for the reader, we give each of these implementation a reference name, a pointer to the relevant section of the paper and a short description.

PURE-DEEP. See $\$ 5.1$ A pure Coq implementation of BDDs that makes a deep embedding of memory as finite maps and uses indices as surrogates of pointers. PURE-Shallow. See §5.2. A pure Coq implementation of BDDs that uses a shallow embedding of memory.

SMART. See $\$ 6.1$. An "impure" implementation of BDDs in Coq: we implement hash-consing and memoization through the extraction mechanism of Coq.

SMART+UID. See $\S 6.2$. A variation on the previous approach in which we discuss how to expose and axiomatize the operations on the unique identifiers associated with BDD nodes.

\section{Pure solutions}

As the reader can gather from the summary above, we describe two pure Coq implementations of BDDs libraries in this section, that differ mainly by the way we model the memory and the allocation of nodes.

\subsection{The PURE-DEEP approach}

The idea here is to model the memory using finite maps inside Coq and use indices in the maps as surrogates for pointers, implementing BDD operations as manipulations of these persistent maps. Such an implementation ${ }^{4}$ was described in 22,23 , but we propose a new one here for the sake of completeness, and because the old one did not age well w.r.t. the evolution of Coq. Our implementation is defined as follows.

\footnotetext{
4 One should also mention the seminal work by E. Ledinot in 1993 on the canonicity of binary decision dags, available from the Coq contributions.
} 
First, we assign a unique identifier to each decision node. Second, we represent the directed acyclic graph underlying a BDD as a Coq finite map from identifiers to decision nodes (that is, tuples that hold the left child, the node variable and the right child). For instance, the following graph, on the left, can be represented using the map on the right.

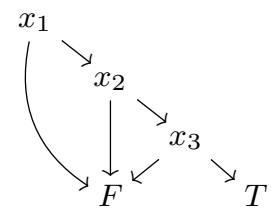

$$
\begin{array}{ll}
1 & \mapsto \quad\left(\mathrm{F}, x_{1}, \mathrm{~N} 2\right) \\
2 & \mapsto \quad\left(\mathrm{F}, x_{2}, \mathrm{~N} 3\right) \\
3 & \mapsto \quad\left(\mathrm{F}, x_{3}, \mathrm{~T}\right)
\end{array}
$$

Then, we implement the hash-consing pool using another map from decision nodes to node identifiers and a next counter that is used to assign a unique identifier to a fresh node. In the situation above, next is equal to 4 and the hash-consing map is defined as follows

$$
\begin{array}{cl}
\left(\mathrm{F}, x_{1}, \mathrm{~N} 2\right) & \mapsto 1 \\
\left(\mathrm{~F}, x_{2}, \mathrm{~N} 3\right) & \mapsto 2 \\
\left(\mathrm{~F}, x_{3}, \mathrm{~T}\right) & \mapsto 3
\end{array}
$$

Notations. Through this paper, we stay as close as possible to our Coq code. Yet we allow us some liberty when it comes to finite maps (that are pervasive in our code): the notation $t \rightsquigarrow s$ denotes a type of efficient maps from type $t$ to type $s$; and we use indiscriminately the notations get and set that respectively access and update such finite maps. Implicitly, these two notations have the following types:

$$
\begin{array}{ll}
\text { get } \quad: \quad \mathrm{A} \rightarrow(\mathrm{A} \rightsquigarrow \mathrm{B}) \rightarrow \text { option } \mathrm{B} \\
\text { set } \quad: \quad \mathrm{A} \rightarrow \mathrm{B} \rightarrow(\mathrm{A} \rightsquigarrow \mathrm{B}) \rightarrow(\mathrm{A} \rightsquigarrow \mathrm{B})
\end{array}
$$

(Note that we do use the efficient finite maps from the Coq standard library; but we chose here to abstract from the particular module definitions and names that we have to use in our code, for the sake of legibility.)

For the practical mind. In the following, we use Coq's positive numbers positive as unique identifiers, and use Patricia trees for finite maps.

Fig. 3 shows our inductive definitions and the code of the associated allocation function mk_node, knowing that alloc $\mathrm{n}$ st allocates the fresh node $\mathrm{n}$ in the hashconsing state st (taking care of updating both finite maps and incrementing the "next fresh" counter). Then, equality between BDDs (eqb) is provided by decidable equality over node identifiers.

All operations thread the current global state in a monadic fashion. The correctness of BDD operations corresponds to the facts that 1) the global state is used in a monotonic fashion (that is the structure of the resulting global state is a refinement of the input one, see Fig. 4);2) the resulting global state is well-formed; 3 ) the denotation of the resulting BDD expression is correct.

Invariants and well-formedness properties. We present the well-formedness properties we preserve over expressions (wf_expr) and over hash-consing states (wf_hashcons) in Fig. 5. The inductive predicate wf_expr st $\mathrm{v}$ e depends on a variable level $\mathrm{v}$ that indicates a bound over the variable identifiers appearing in the BDD e. This ensures 


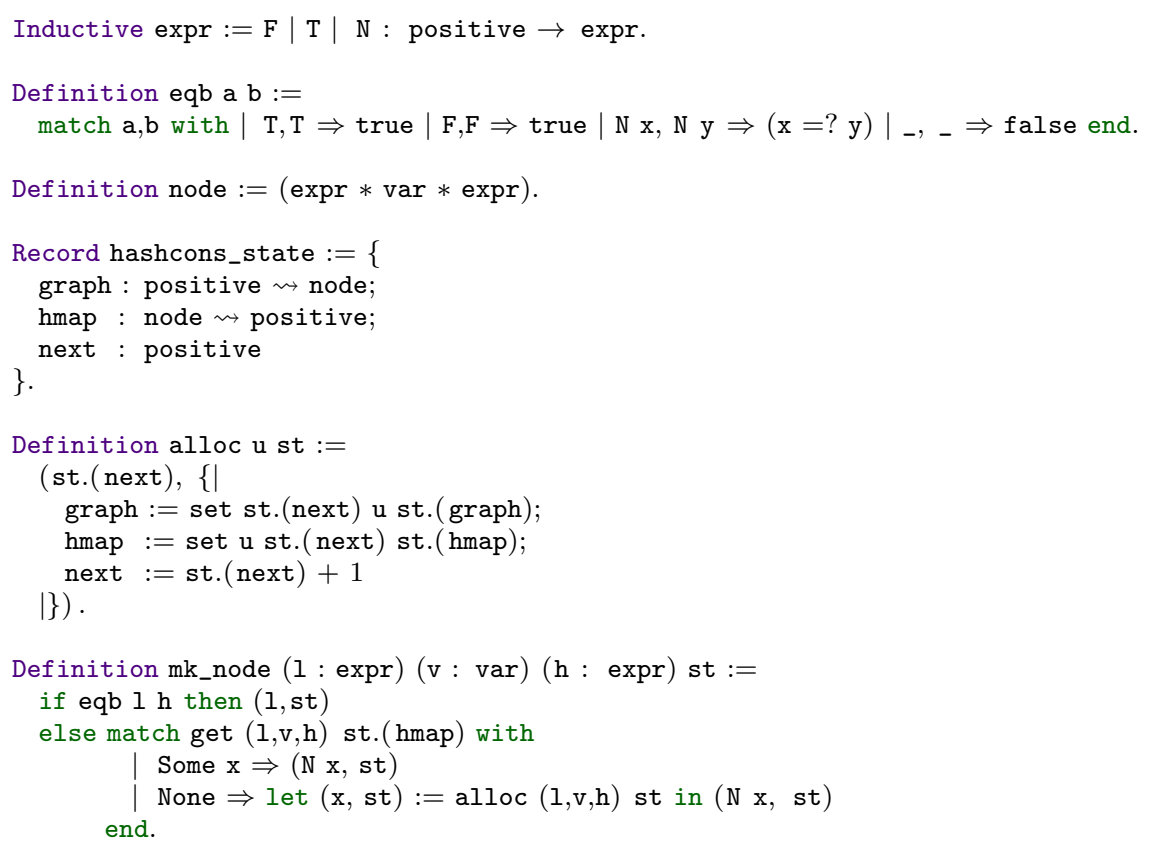

Fig. 3: Hash-consing in pure Coq, using a deep-embedding

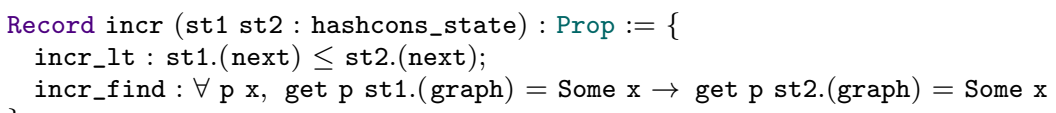

Fig. 4: Monotonicity predicate over hash-consing states

that variable are kept ordered. Then, a well-formed expression is either a leaf (cases wf_T and wf_F) or a pointer to a hash-consing node with a head variable w less than the bound v. (Remark that this definition of well-formed expression is not directly recursive, because the actual recursion takes place in the definition of wf_hashcons below; this notion of well-formed expressions coupled with well-formedness of the state suffice to prove that expressions are DAGs with a suitable ordering on the variables.)

The predicate wf_hashcons is the general well-formedness property over hashconsing states. The predicate wf_bijection ensures that the two maps hmap and graph form a bijection; wf_expr_lt_next ensures that the next fresh variable counter will indeed produce fresh variables; wf_map_wf_expr_l and wf_map_wf_expr_r ensure that a node stored in the hash-consing structure has well-formed children, respecting the variable order; wf_reduced ensures that all those nodes are reduced (ie. their left child is different from their right child). 


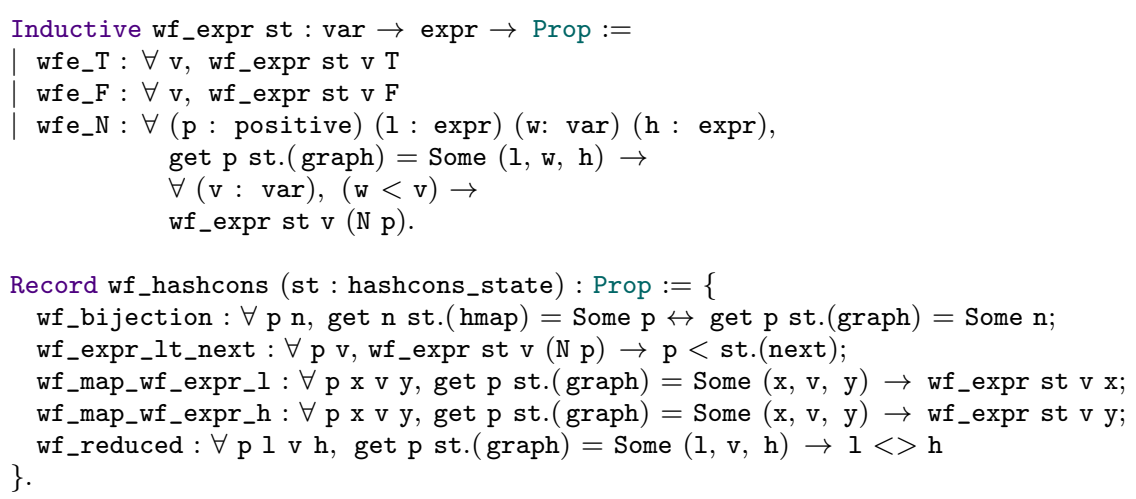

Fig. 5: Well-formedness of hash-consing state

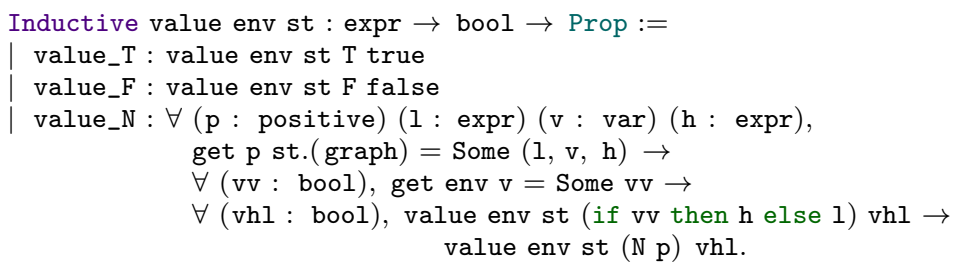

Fig. 6: The denotation of BDD expressions

Note that the statement that the hash-consing structure is correct corresponds to wf_bijection and wf_expr_lt_next. The other statements correspond to properties about BDDs, namely the facts that they are reduced and ordered.

The denotation of BDD expressions. Recall that BDD expression, as we have defined them in this section, do not have an inductive structure we can follow: this means that we cannot define the semantics of BDD expressions as a Coq fixed-point. Rather, we have to define the semantics of BDD expressions as an inductive predicate that uses the state of the hash-consing data structure to go through the graph. The inductive value is shown on Fig. 6. It is defined as a binary relation with two parameters (the valuation of variables env and the state of the hash-consing structure st) and two arguments: the expression and its denotation (a Boolean).

For the practical mind. We only use the type of environments env to establish a semantics, thus efficiency is not an issue. However, we prefer to use finite maps of some kinds to represent environments: using a function of type var $\rightarrow$ bool would force the client to reason about the fact that some BDD expressions are insensitive to the valuation of some variables. Using a finite map make this reasoning internally in our library and eases the client's life.

Termination of $B D D$ operations. The first problem that we have to solve in our Coq representation is that, as can be expected from our data structure, BDD operations 


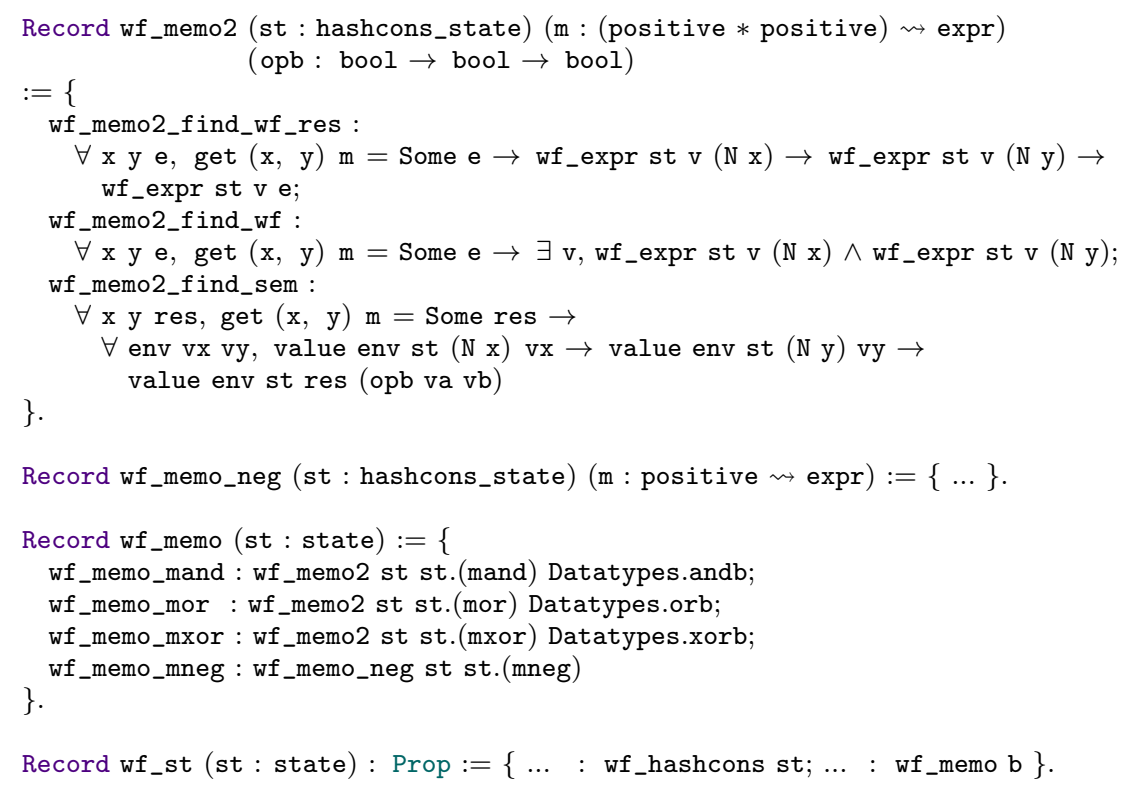

Fig. 8: Invariant over the memoization information

(Note that finding the correct pattern of memoization for a program is still an art rather than a science: using the data structure above, we keep the memoized values from one run of an operation to the other. In this section, we will settle on this conservative strategy, but other memoization strategies are possible that yield different performance and memory consumption profiles.)

A mouthful of code. The final version of our code is shown on Fig. 9. We use a do-notation à la Haskell to make it more palatable.

Then, we prove that under some hypotheses, this combinator is correct: that is, it produces well-formed hash-consing structures and memoization tables, and the denotation of the resulting expression is correct. For the sake of clarity, we will not expose these hypotheses nor the resulting correctness theorem in this paper, and refer the interested reader to the archive of code that accompanies this paper [5].

Implementing the and operation. However, we demonstrate the use of this combinator on the particular example of the and function; all other binary operations follow the same pattern. First, we have to define a function upd_and that updates the memoization state: it is simply a wrapper that add an element to the right memoization table, and leave the others untouched. Then, we define the function mk_and as a simple call to the binary combinator. The crux here is the choice of the functions $\mathrm{Fx}$ and $\mathrm{Tx}$ that specify the behavior of the combinator at the leaves of the DAG. 


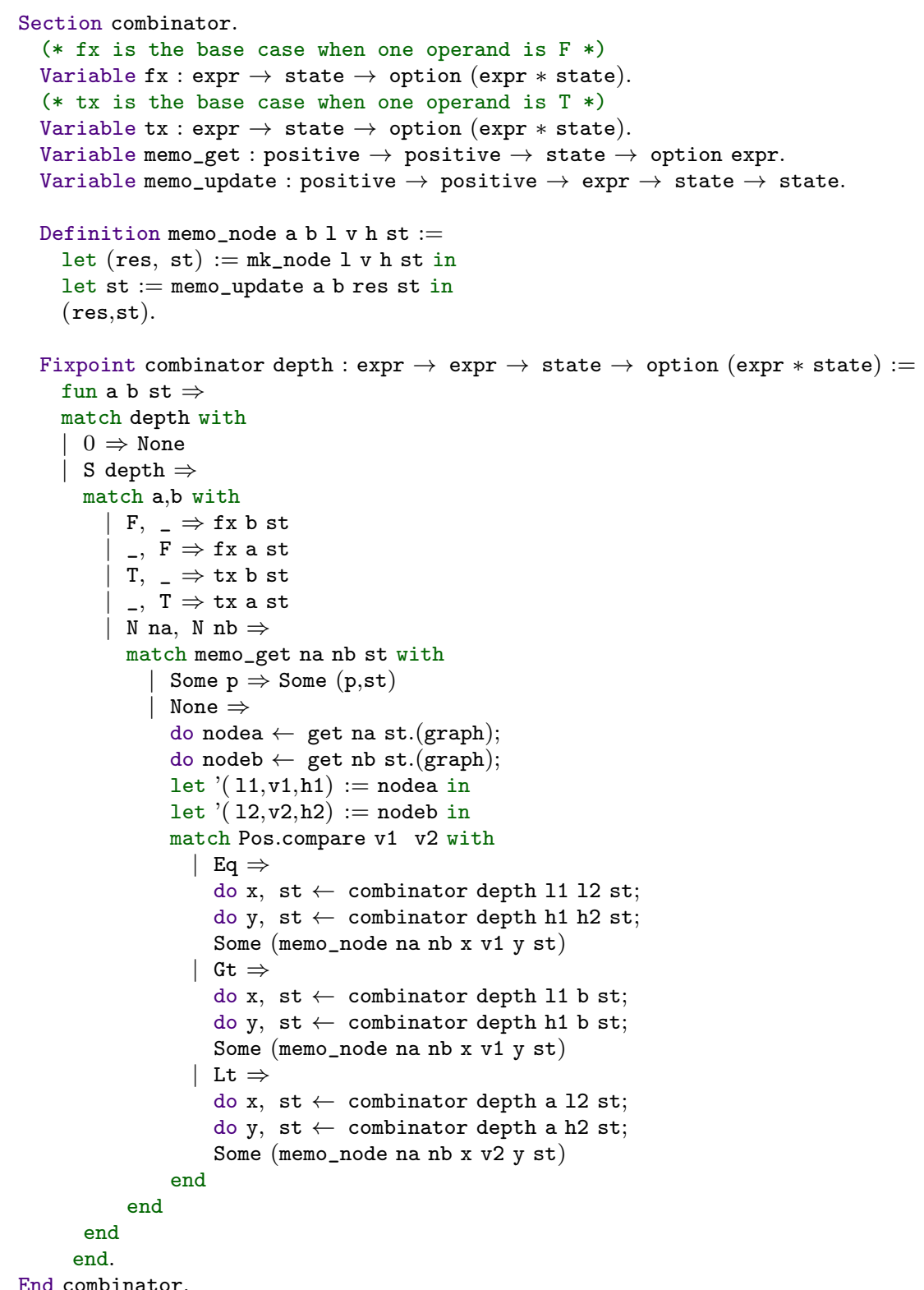

Fig. 9: Deep combinator for binary operations 
with termination. Therefore, we would like to be able to reason about BDDs as if they formed an inductive type, while keeping the ability to share sub-terms at runtime.

There is actually no need to look further than inductive types to do that. The standard intuition about inductive types is that they define the smallest type closed under application of constructors: the mental image that we get from that is a tree. Yet, there is nothing that prevent us to use the system to share sub-terms

In this section, we demonstrate that we can encode BDDs in Coq using a representation that looks like binary decision trees, yet has runtime performances similar to the PURE-DEEP implementation (see \$5.1), using explicit sharing. We present on Fig. 10 our inductive definitions, and the associated allocation function mk_node. This has to be compared with Fig. 3: the difference mainly lies in the deletion of the graph map, and the explicit recursive structure of the expr type.

For the practical mind. The reader may wonder why we chose to define expr as two mutual inductive types expr and hc_expr. Indeed, we explain in $\S 8.3$ that it is possible to inline hc_expr inside the definition of expr. The mutual inductive solution is inspired by the hash-consing library in OCaml by Conchon and Filliâtre 9]. This library makes a clear distinction between hash-consing nodes (the equivalent of our hc_expr inductive) and the actual values that are hash-consed. In Coq, this makes some inductive proofs a little bit more involved: we need to use mutual induction on the two data-types.

The code of the hc_node function is subtle: a call to hc_node e bdd will perform a lookup in the hash-consing map hmap: if the same expression was previously allocated, then we return the old version; otherwise, we update the map hmap with a mapping from the expression to its hash-consed version. The lookup ensures that equal expressions (modulo the comparison function used to index hmap) are mapped to the same hash-consed expression. Then, $\mathrm{mk}_{-}$node $\mathrm{l} \mathrm{v} \mathrm{h}$ bdd will first test the identifiers of 1 and $\mathrm{h}$ for equality: if it is the case, then there is no need to introduce a new node; otherwise, we perform a call to hc_node.

As an example, assume that $\mathrm{x}$ and $\mathrm{y}$ are expressions with different identifiers. The following code

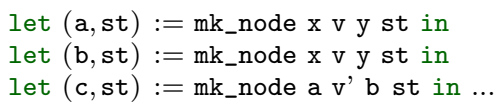

will make $\mathrm{a}, \mathrm{b}$ and $\mathrm{c}$ point to the same memory location! However, if $\mathrm{x}$ and $\mathrm{y}$ were not shared maximally, then neither are a, b nor c.

A word on memoization. There is no difference at all in the way we handle memoization in this implementation w.r.t. 55.1 . That is, we implement the same state record as before; and pass the same memoization tables around.

\footnotetext{
6 Such an approach may be interesting even if each structure is a tree, but different trees can share sub-trees. For instance, the Astrée static analyzer implements maps as balanced binary trees with opportunistic sharing 3 3 $§ 6.2]$ : e.g. when an idempotent operation $f(x, x)=x$ is to be performed on the images of two maps $g$ and $h$, returning the map $x \mapsto f(g(x), h(x))$, then if $g$ and $h$ are determined to be identical through pointer equality, $g$ is directly returned.
} 


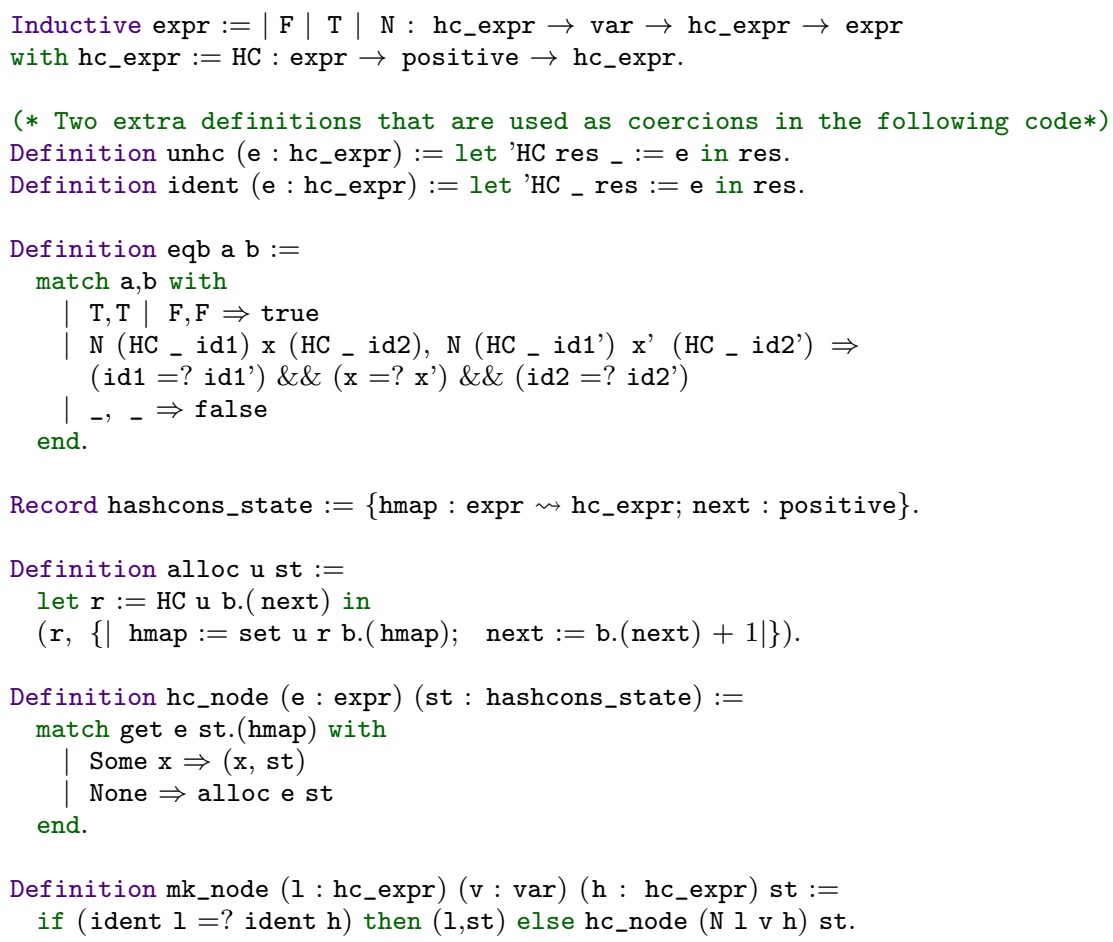

Fig. 10: A shallow-embedding of sharing

A word on termination. It is now easier to define recursive functions that operate on BDDs by taking advantage of the inductive definition of expr. We have to stress that easier is not easy because the Coq termination checker requires that recursive calls are made on a structurally smaller argument: there is no built-in support for recursive definitions with pairs of arguments that are decreasing w.r.t. a lexicographic order. Therefore, we have to use nested fixed-points or prove that the recursive calls are well-founded. In this section, we choose the former.

Re-implementing the combinator. We are now ready to describe the code of the implementation of the binary combinator presented in Fig. 11. The code is similar to the one in Fig. 9 with a few key differences: thanks to the inductive definition of expressions, we do not have to perform lookups in the graph map and we do not use fuel anymore. This makes the combinator function total; and we can get rid of the Maybe monad.

Canonicity. Again, we prove that this representation of BDDs is canonical: wellformed equivalent expressions are mapped to the same nodes. Again, we have the corollary that the (non-recursive) equality test from Fig. 10 that inspects the (top-level) node identifiers is a complete and correct characterization of semantic equivalence. 


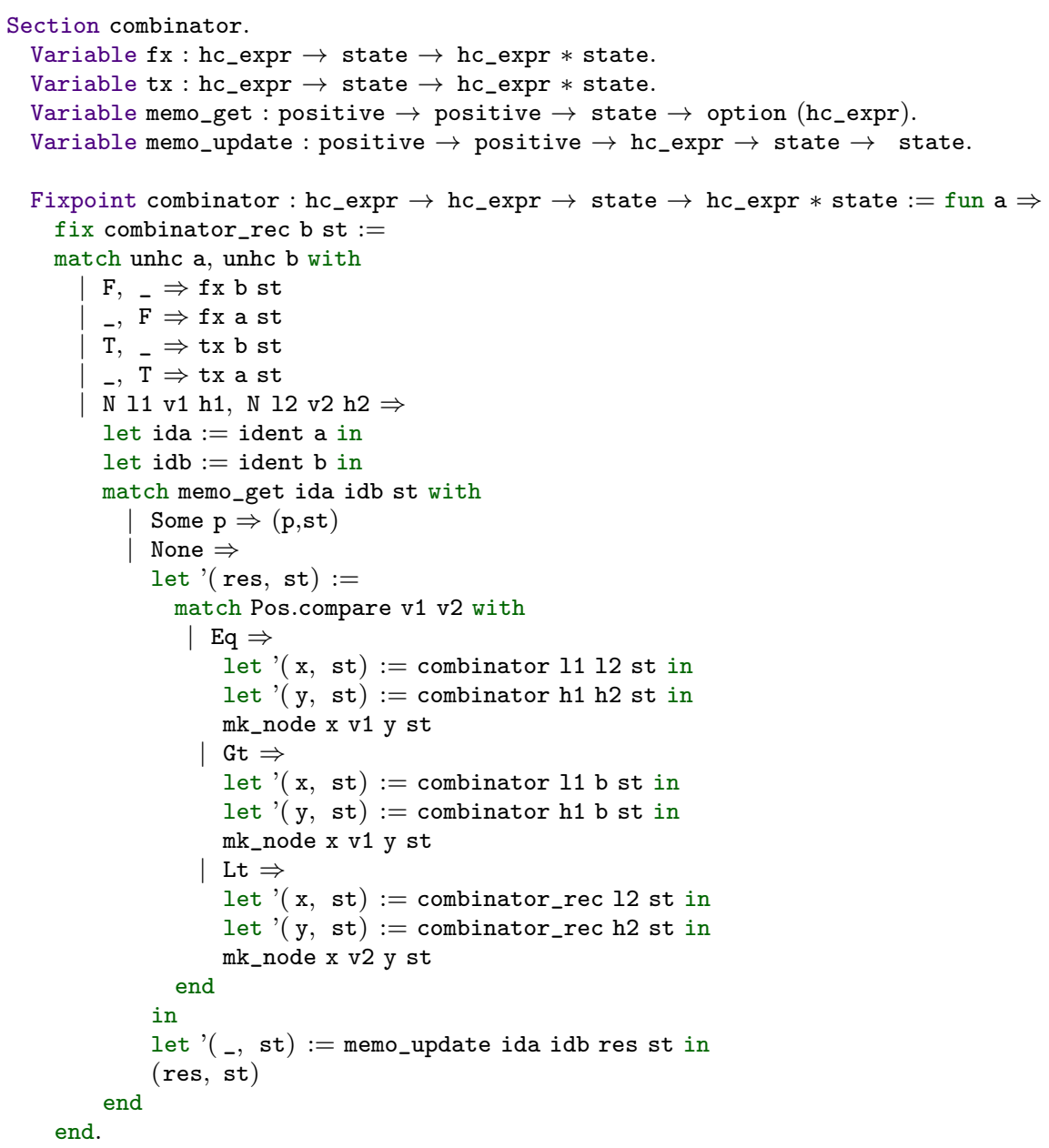

End combinator.

Fig. 11: Shallow combinator for binary operations

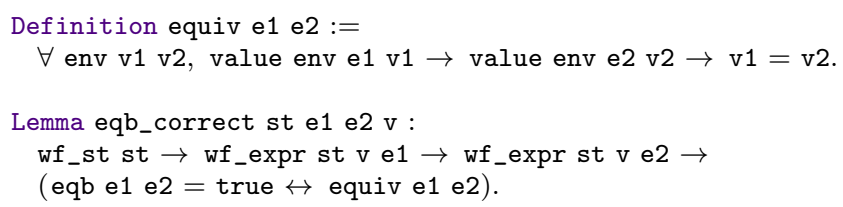

Comparison with the previous approach. The implementation presented in this section is derived from the previous one, with the following improvements: the proofs are roughly $20 \%$ shorter; the performances are slightly better when executing the code inside Coq (there is less administrative book-keeping to do in our data structures); the functions that operates on BDDs are total. Furthermore, it would 
probably be easier to implement garbage collection in this setting than in the previous one, thanks to the simpler definition of the global state.

The situation is almost ideal for the equality test: we prove that the (nonrecursive) equality function that inspects the top-level identifiers of nodes is a correct and complete characterization of semantics equivalence of BDD expressions. However, we have no way to prove that it corresponds to physical equality. Actually, we cannot state within Coq that it is never the case that two identical representations of the same term coexists, even if we could argue at a meta-level that it is indeed not the case.

\section{From pure data structures to persistent data structures via extraction}

In the previous section, we use a state monad to store information about hashconsing and memoization. However, one can see that, even if these programming constructs use a mutable state, they behave transparently with respect to the pure Coq definitions.

We have seen earlier (see $\$ 2.3$ that if we abandon (efficient) executability inside Coq, we can express new idioms. In the following, we implement the BDD library in Coq as if manipulating decision trees with neither sharing, nor hash-consing tables, nor memoization tables, then add the hash-consing and memoization code by using special features of the extraction mechanism to remap some constants arbitrarily to custom OCaml code.

\subsection{The SMART approach}

More precisely, we define our BDDs as binary decision trees (see Fig. 12a), and implement operations in Coq on this simple data structure. Then, we tell Coq to extract the bdd inductive type to a custom bdd OCaml type (see left of Fig. $12 \mathrm{~b}$ and to extract constructors into smart constructors that maintain the maximal sharing property. The type defined in OCaml is identical to the Coq one, except that it carries one extra field of type tag, morally containing the associated unique identifier. The smart constructors make use of the hash-consing library used in Why3 20], a recent version of a library by Conchon and Filliâtre [9]. It defines the Hashcons.Make functor, that we instantiate. The generated module provides a HCbdd.hashcons function that returns a unique hash-consed representative for the parameter.

The reader may notice that we choose to name bdd in Coq what is clearly a representation of a binary decision tree, and which corresponds to what was previously named expr. We believe that this particular choice of name makes sense if we consider values of type bdd to represent Boolean functions.

Discussion: the status of the equality test. In Coq, we define the obvious recursive function bdd_eqb of type bdd $\rightarrow$ bdd $\rightarrow$ bool, that decides structural equality of BDDs. Then, we extract this function into OCaml's physical equality:

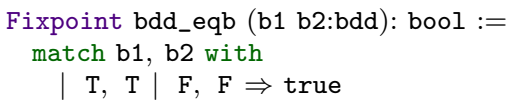




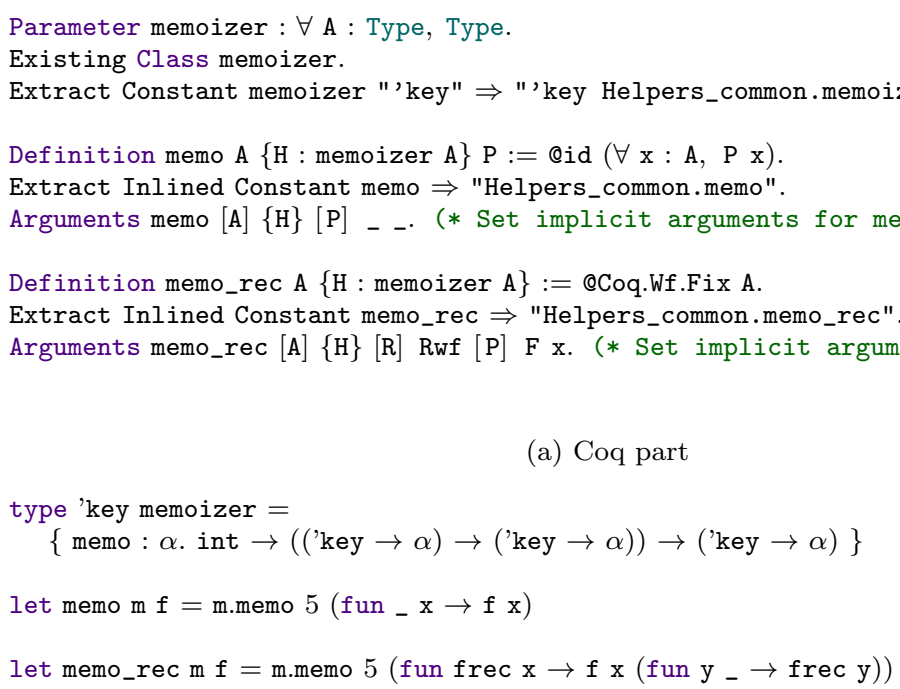

(b) OCaml part

Fig. 13: Memoizing combinators

Note that the user is forced to use this function instead of using the $\mathrm{N}$ constructor ${ }^{7}$ Failing to do so will result in code that is correct, but does not build reduced binary decision diagrams. More precisely, extracting eqb to $==$ would make it possible to prove that BDD operations are semantically correct, but this would make diagrams non-canonical: well-formed expressions could be represented by several different nodes.

Implementing the combinator. The last ingredient needed to transform a decision tree library into a BDD library is memoization. We use the same kind of ideas: we define our functions as if not memoized, but we use a special well-founded fixed-point combinator, that we extract to a memoizing fixed-point combinator. The details can be seen on Fig. 13, we declare an abstract type class memoizer of types A such that we know how to memoize functions of type $\forall \mathrm{x}: \mathrm{A}, \mathrm{P} \mathrm{x}$. This is extracted in OCaml to the type of polymorphic fixed-point combinators, with an extra technical int parameter used to specify the initial size of the used hash map. In Coq, we then define a memoizing combinator memo and a memoizing fixed-point combinator memo_rec as if they were not using memoization, but we ask the extraction mechanism to map them to special OCaml functions, that make use of the type class instance given as parameter. These functions are observationally equivalent to the Coq ones, provided that the type class instance is correct, and that the memoized function is pure.

It is worth noting that directly exposing the type 'key memoizer in Coq would be unsound, because this allows to use its instances in a non-terminating manner:

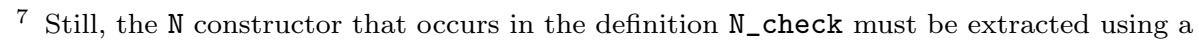
smart-constructor. 


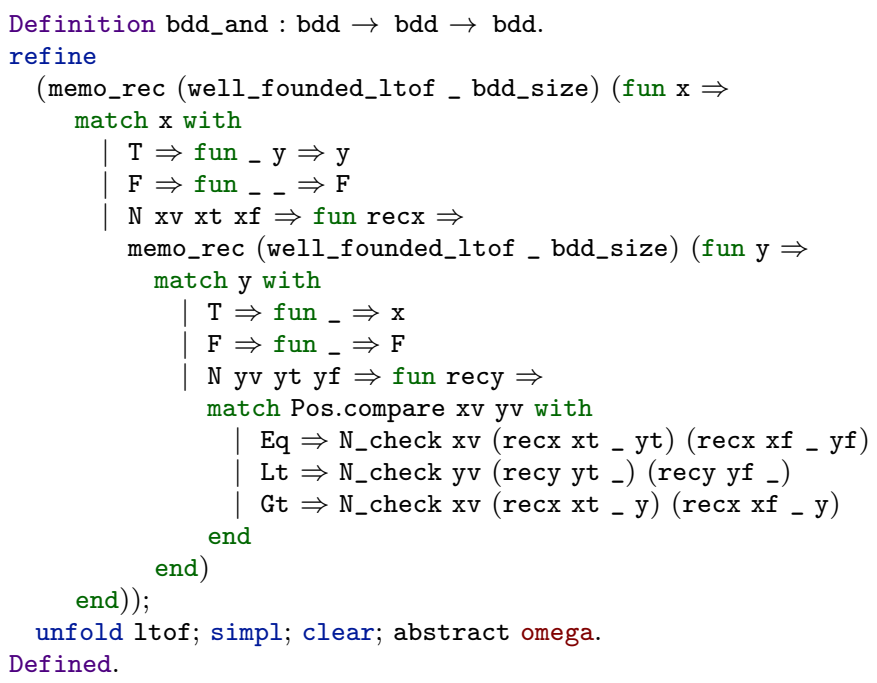

Fig. 15: The and operation on bdds

accessible after the function call, so that the garbage collector is going to collect most of the memoized data at each cycle. Using our pattern, we make sure a memoized datum is not reclaimed as long as both parameters are still alive in memory. (Note that if we choose to use regular hash-tables instead of weak ones, this argument does not stand anymore, but the program has a different performance and memory consumption profile.)

Discussion: comparison with previous approaches. In this instance of our BDD library, all Coq definitions are kept simple and proofs are straightforward. That is, we can prove semantic correctness of all operations directly using structural induction on decision trees and there is no state holding structures. There is a nice separation between the hash-consing and memoization code, that is generic, written in OCaml and not proved and the BDD code, mostly written and proved in Coq. We discuss the lack of confidence in the OCaml code and how it can be restored in 9.1 .

We do not need to use monads in the Coq code, so that the interface of the BDD library remains modular and easy to use. Moreover, it is straightforward to implement garbage collection strategies in order to avoid memory leaks.

\subsection{The SMART+UID approach}

The previous SMART approach totally hides the unique identifiers from the Coq code. Yet, exposing these unique identifiers may be useful at times.

Consider the following use case: from a BDD $B$ we would like to build an equivalent propositional logic formula of linear size, for instance for feeding into a satisfiability modulo theory solver. In order to avoid an exponential blow-up, each shared sub-tree should generate one single sub-formula, used in a "let" binder so 
that its value can be used in multiple occurrences. The obvious way to implement such a transformation is to first detect which sub-trees are shared, using a set of shared subtrees seen so far, then to perform the transformation, using a table of mappings from subtrees to bound variables.

It seems therefore highly desirable to be able to build sets and maps over our hash-consed type. Generic functional sets and maps are usually implemented using balanced trees over a totally ordered data type; for efficiency, the comparison function should be very fast. An obvious choice would be to expose the unique identifiers to the Coq code (through a function bdd $\rightarrow$ uid), or at least the total order that they induce (through a function bdd $\rightarrow$ bdd $\rightarrow$ comparison).

Unfortunately, doing so without precautions can lead to unsoundness. Consider a program where, in succession, two nodes $A$ and $B$ are allocated, then a node $A^{\prime}$ isomorphic to $A$ is created; let $u_{A}<u_{B}, u_{A^{\prime}}$ be the successive unique identifiers. If $A$ is collected between the allocations of $B$ and $A^{\prime}$, then $A^{\prime}$ will be allocated, with $u_{A^{\prime}}>u_{B}$. Yet, $A^{\prime}$ and $A$ are, from the point of view of the Coq code, identical; thus $u_{A^{\prime}}=u_{A}$, yielding an inconsistency.

The workaround is to use a normal hash-table, as opposed to a weak hash-table, which prevents the collection of unreachable nodes. Then, two identical nodes created within the same execution are necessarily physically equal and thus share the same identifier.

One difficulty remains. Gallina is a purely functional language; the evaluation of a given term always yields the same result, and one expects the same property to extend to the extracted OCaml program, as long as it does not interact with the external world (e.g. reading from files). Yet, this is not necessarily the case if one exposes the unique identifiers. Consider a program $P_{1}$, such that the extracted OCaml code allocates two nodes $A$ and $B$ in this order. If $P_{1}$ is run stand-alone, then $u_{A}<u_{B}$. Yet, if another program $P_{2}$ allocating $B^{\prime}$ isomorphic to $B$ is first run, and $B^{\prime}$ is not collected, then $B^{\prime}$ is the same as $B$ and $u_{B}=u_{B^{\prime}}<u_{A}$.

It seems questionable that the result of an evaluation should depend on whether or not some other (unrelated) evaluation has taken place, if only because it makes debugging difficult. 8

\subsubsection{Maps and sets over hash-consed types}

Arguably, the final result of an algorithm using functional sets and tables should not depend on the order relation used. One could thus opt to expose neither the unique identifiers, nor the order relation they induce, but only a functional map interface (with find, remove and update). Then, the results cannot depend on garbage collection and other disturbances to the ordering. Yet, one could also want these sets and maps to provide a fold operation; this would need to be restricted to associative and commutative operations (so that their result do not depend on

8 One could argue that, with certified programs in the Coq fashion, there is no need to debug: each function or module comes with a proof of its correctness, which compositionally entail the correctness of the whole program. Yet, commonly one only proves the results to be correct, not necessarily optimal, and one proves very seldom that the computation has the expected complexity. Furthermore, some computations are split between an untrusted solving procedure, and a trusted checker; a failed check entails having to debug the untrusted procedure, which may be hard if behaviors are hard to reproduce independently of the rest of program. 
mentioned limitation applies: since unique identifiers are visible, one cannot use weak hash tables and the OCaml garbage collector.

The use of axioms is debatable. On the one hand, the use of axioms somewhat lowers the confidence we can give in the proofs, and they make the code not executable within Coq. On the other hand, these axioms are actually used implicitly when extracting Coq constructors to "smart constructors": they correspond to the meta-theoretical statement that these constructors behave as native Coq constructors. Thus, they make explicit some of the magic done during extraction.

We could gain confidence about the fact that our axiomatization is consistent, by using Coq's module system. That is, we could parameterize our development in this section by a suitable interface specifying the various functions that we axiomatized. Then, we could instantiate this interface either with axioms, and extract them to an efficient yet unproven implementation in OCaml; or, we could instantiate them with a pure Coq version. This would prove that this set of axioms is not unsound in Coq. This general approach was used by Vafeiadis 21 to prove that his interface for adjustable references is sound, and we could do the same here. We have yet to conduct this experiment.

\section{Discussion}

In this section, we compare our design patterns on various aspects. Let's start with the easy ones:

Executability inside Coq. The PURE-DEEP and PURE-SHAllow implementations can be executed inside Coq, and have decent performances. The SMART approach can also be executed inside Coq, but has dreadful performance (when executed inside Coq, it uses binary decision trees). The SMART+UID approach cannot be executed inside Coq.

Trust in the extracted code. Unsurprisingly, the SMART and the SMART+UID approaches yield code that is harder to trust, while the PURE-DEEP and PURESHALLOW approaches leave the extracted code pristine.

Proof. From a proof-effort perspective, the SMART approach is by far the simplest. The SMART+UID approach involves the burden of dealing with axioms. However, it makes it easier to trust that what is formally proven corresponds to the real behavior of the underlying runtime. By comparison, the PURE-DEEP and PURESHALLOW approaches required considerably more proof-engineering in order to check the validity of invariants on the global state. Note however that our proof arguments are much simpler in the latter one.

Garbage collection. Implementing (and proving correct) garbage collection for the PURE-DEEP Or PURE-SHALLOW approaches would require a substantial amount of work. By contrast, the SMART approach make it possible to use OCaml's garbage collector to reclaim unreachable nodes "for free".

Operations As we have shown, binary operations can be handled with a single parametric combinator. All our implementations use such a combinator to implement conjunction, disjunction, exclusive-or and so on. There is little work to do to add support for any binary operation we may have overlooked. The situation is more complicated when it comes to ternary operations (such as the if-then-else Boolean operation). Implementing it using the SMART approach, 
and proving it correct requires around 80 lines of code. It would require a nontrivial amount of work to implement it using our PURE-DEEP or PURE-SHALLOW approaches, and we have not conducted this experiment yet. Other operations that are relevant in a BDD library are function composition and quantification. We have yet to implement these in any of our libraries. Quantification over one variable can be defined as a structural recursion over one BDDs. As such, we think it can be easily defined in all our frameworks. Unary composition (that is, substitution of one variable with another Boolean function) can be defined as a structural recursion over two BDDs. As a consequence, implementing it in the SMART framework seems simple. However, this particular recursion scheme does not fit the combinator of the PURE-DEEP or PURE-SHALLOW approaches, and some additional work would be needed in order to implement it using those approaches. Moreover, a non-negligible amount of work would be needed to support variable arity composition and quantification in all libraries.

\subsection{Performances of the extracted code}

We evaluate the performances of the OCaml code that is extracted from our "pure" (see \$5) and "impure" (see §6) libraries, and we pit them against a reference library implemented in OCaml (available from J.C. Filliâtre's web page). This reference library does not keep the memoization table alive from one execution of an operation to another: for instance, each time the and function is called, a new memoization hash-table is allocated. Therefore, to make up for a fair benchmark with our implementations, we modified this reference library to use a memoization strategy closer to ours. This alternative reference implementation is designed "reference (conservative)" in what follows.

Then, this evaluation, we use two standard benchmarks (see 23]). The first one is Urquhart's formula $U(n)$ defined by

$$
U(n) \triangleq x_{1} \Longleftrightarrow\left(x_{2} \Longleftrightarrow \ldots\left(x_{n} \Longleftrightarrow\left(x_{1} \Longleftrightarrow \ldots\left(x_{n-1} \Longleftrightarrow x_{n}\right)\right)\right)\right)
$$

The second kind of formula states the pigeonhole principle $P(n)$ for $n+1$ pigeons: that is, if there is $n+1$ pigeons in $n$ pigeonholes, then there is at least one hole that contains two pigeons. The plots of the execution time required to check that these formulas are tautology are given in Fig. 17.

First, we remark that the reference implementation that we use is (almost always 4 times faster than our best implementation SMART. There is no significant difference in execution time between the reference implementation with the conservative memoization strategy and the reference implementation. We have not conducted a detailed measurement of the memory consumption ${ }^{11}$ though, and the memory consumption profile is likely to be different between the two.

Then, our SMART implementation is roughly 4 times faster than the PURE-DEEP one: interestingly enough this value is consistent over our benchmark, while we

\footnotetext{
10 This is not necessarily true for small formulas due to differences in the start-up costs of these libraries.

11 These particular benchmarks were run on a computer with 1 TB of ram, which made it possible to avoid swapping during our experiments. Indeed, memory usage is the limiting factor on bigger instances of the tests.
} 


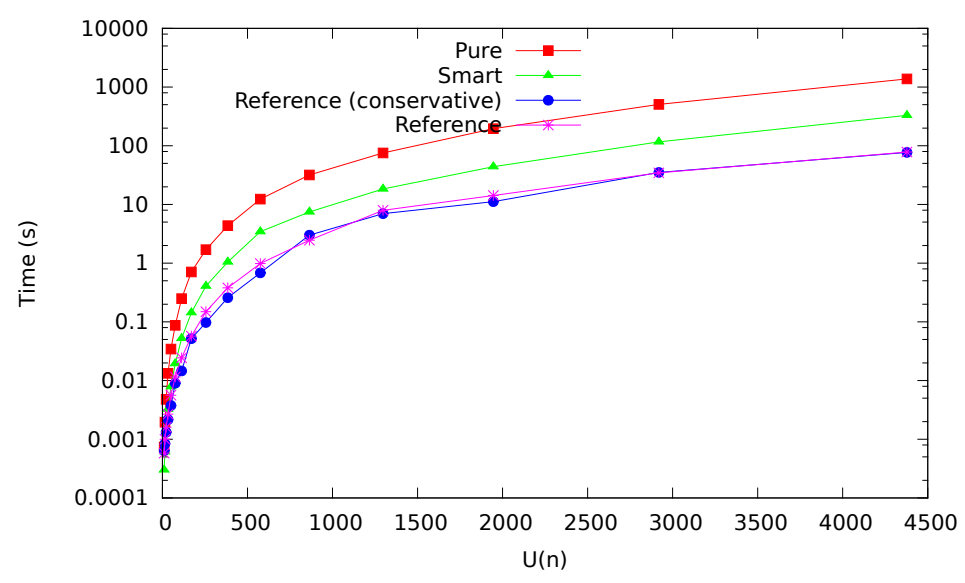

(a) Urquhart's formula

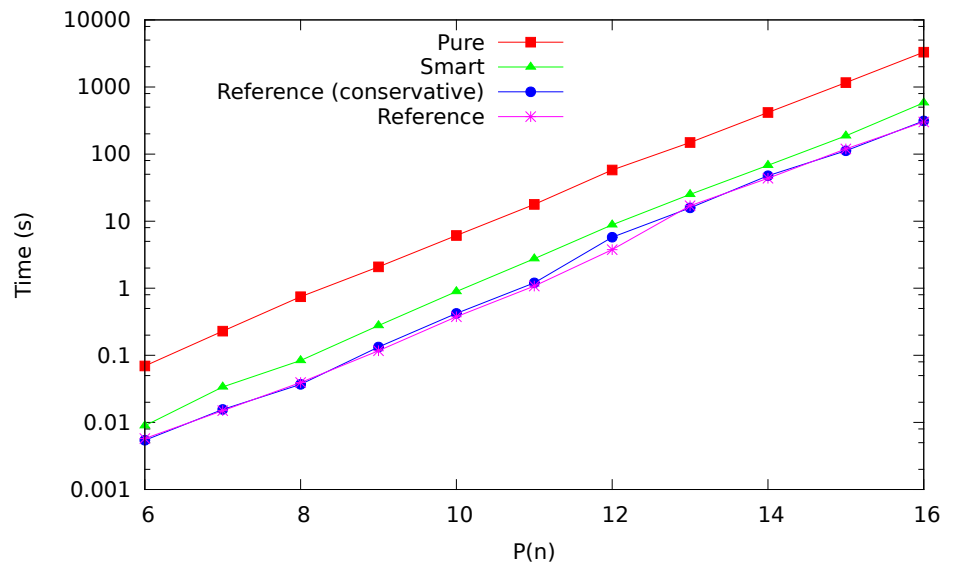

(b) Pigeonhole principle

Fig. 17: Execution time for the BDD benchmarks

could have expected logarithmic factors to show up. Indeed, recall that the pure implementation uses functional finite maps, while the smart one uses OCaml hash-maps.

Also, we have run the same benchmark on our PURE-SHALLOw implementation, and the resulting plot is close to the PURE-DEEP plot: for small instances, the difference of execution times are of about $10 \%$. For our largest instance of the pigeonholes problem, we observe that the PURE-SHALlOw is faster by about $30 \%$. We do not delve too much on this result: we wonder for instance to what extent changing the implementation of finite maps that we use could result in similar differences of performances.

Primary investigation show that the main performance difference between the reference implementation and our SMART approach come from the use of the generic hash-consing library: the reference library uses a specialized hash-consing system, 
much faster than the generic library we use. Similarly, the difference between the SMART and the PURE-SHALLOW seems to be the use of AVL trees to represent dictionaries.

We did not investigate much the memory behavior. Our simple experiments show that it depends on the benchmark we consider. The bottom line is that all our approaches consume less than twice as much memory as the reference implementation, conservative version. Moreover, we observe that PURE-SHALLOW consumes 10\%-20\% less memory than PURE-DEEP. Concerning the SMART approach, it seems like our Curryfied memoization scheme creates a lot of very small hashtables in the OCaml heap, which is not the optimized use case of OCaml hash-tables. Thus, there might be room for improvement here.

\section{Implementing reduction in the $\lambda$-calculus}

In the previous sections, we have presented several design patterns about how to implement hash-consed data structures in Coq. In this section, we apply two of them to the example of reduction of $\lambda$-terms (the running example of Conchon and Filliâtre 9]).

\subsection{An implementation without hash-consing}

We first describe a Coq implementation, without memoization nor hash-consing, of $\lambda$-terms with de Bruijn indices inspired from Huet's Constructive Computation Theory [14. We implement four functions on terms: lift, subst, hnf (that puts a term in head-normal form) and $\mathrm{nf}$ (that puts a term in normal form). This amounts to roughly 60 lines of Coq code: we present the code for lift in Fig. 18b as a reference for future comparisons.

Then, we reuse Conchon and Filliâtre's benchmark 9]: we implement a $\lambda$ calculus version of quicksort that operates on lists (encoded as $\lambda$-terms) of Church numerals; and apply this algorithm to sort the list $L=(0:: 3:: 5:: 2:: 4$ :: 1 :: nil $)$.

Using Coq's virtual machine, sorting this list requires over $250 \mathrm{~s}$ on a recent desktop computer ${ }^{12}$ this involves a number of reduction that is exponential w.r.t. the size of the list and the size of the numbers. Extracting the code to OCaml makes it more efficient by a constant factor. We compile the extracted code with the native OCaml compiler, and run it to sort the list $L$.

\begin{tabular}{|c|c|c|}
\hline time $(\mathrm{s})$ & Number of allocated bytes & Max. size of the major heap (words) \\
\hline 30 & $47 * 10^{9}$ & $1.1 * 10^{6}$ \\
\hline
\end{tabular}

The number of allocated bytes is given as reported by Gc.allocated_bytes: it corresponds to what is allocated during the execution of the sorting algorithm. The maximum size of the major heap is given as reported by OCaml Gc.stat function. In short, these numbers mean that the program allocates an awful lot of short-lived values.

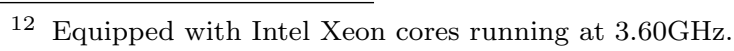




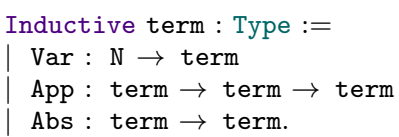

(a) $\lambda$-terms with de Bruijn indices

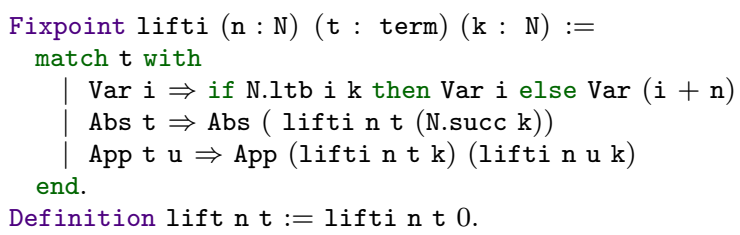

(b) A implementation of lift without hash-consing nor memoization

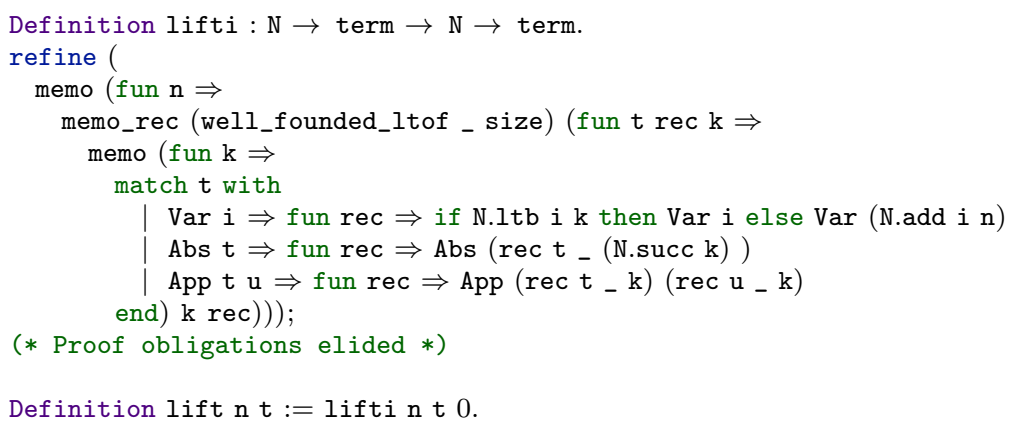

(c) Implementing lift the smart way

Fig. 18: Implementation of terms and lift

\subsection{Using smart constructors}

The SMART approach from $\S 6.1$ involves a small amount of changes to the code, mainly to use memoizing constructs and to add memoizer definitions. To get a rough idea of the changes involved, we present the modified code for lift in Fig. $18 \mathrm{c}$ it is merely a matter of replacing abstractions and fixed-point definitions with their memoizing counterparts. The overall size of the code is now roughly over 80 lines of Coq code. While they do not make it possible to compute efficiently inside Coq, these changes have dramatic consequences on the execution time of the extracted code. Again, we compile the extracted code with the native OCaml compiler, and run it to sort the list $L$.

\begin{tabular}{|c|c|c|}
\hline time $(\mathrm{s})$ & Number of allocated bytes & Max. size of the major heap (words) \\
\hline 0.15 & $0.12 * 10^{9}$ & $3.1 * 10^{6}$ \\
\hline
\end{tabular}

Here, we have a higher top size of the major heap, which account for our use of memoization tables. This is to be weighed against the lower amount of allocated words and the $200 \times$ decrease in running time. 
8.3 Using the shallow-embedding approach

Finally, we use the PURE-SHALLOw from $\S 5.2$. There is now roughly 300 lines of code and definitions to implement lift, subst, hnf and $\mathrm{nf}$. In this section, we will make a brief review of this code, highlighting the differences with respect to what we presented in $\$ 5.2$

We give the modified definition of terms in Fig. 19a; each constructor takes an extra positive argument: that is, we drop the extra indirection we used in the definition of BDD expressions in $\$ 5.2$. (This choice of definition yields simpler proof arguments because we do not need to perform mutual inductions on expr and hc_expr.)

We adapt the notion of global state from $\$ 5.2$. Again, the hmap finite map is indexed using a non-recursive comparison function: it simply compare the head symbols of the terms and, if equal, compare the tags of the sub-terms. Again, we define one memoization table per function we wish to memoize.

Then, let us take the example of abstraction to describe term creation. We define the following smart constructor.

Definition mk_Abs $t$ st $:=m_{k}$ term (Abs t 1 ) st.

The function mk_Abs is a simple wrapper around mk_term: it applies the term constructor Abs to the term $t$ and a dummy unique identifier. Then, mk_term (see right of Fig. 19b performs a lookup in the hash-consing table. If there exists a mapping from Abs $t \mathrm{p}$ (for some $\mathrm{p}$ ) to $\mathrm{x}$ in the table, then we return $\mathrm{x}$. Otherwise, the alloc function replaces the dummy unique identifier with the next fresh unique identifier available, and it updates the map hmap with a mapping from the expression t to its tagged version $r$.

In effect, this code maintains the invariant that all the terms in the hmap table are the canonical representative of their equivalence classes modulo the term comparison used to index hmap. That is, we ensure that the terms we build verify the following property.

Definition wf_term st $t:=$ get $t$ st.(hmap) $=$ Some $t$.

Let us jump to the definition of lifti (see Fig. 19c): it is defined using a wellfounded fixed-point combinator Fixm (elided here) that ensures that the measure $\mathrm{msr}$ is decreasing through recursive calls. The bulk of this definition is lifti_rec: it is quite similar to the previous definitions of lift, except that we have to add fancy return clauses to the match definitions. They are necessary to be able to prove that the measure actually decreases through recursive calls. (Also, note that we could have inlined lifti_rec in lifti, but this two-step definitions makes it for more palatable proof goals later on).

The upside of these definition is that we are now able to compute the normal forms of our $\lambda$-terms inside Coq with decent efficiency: it takes roughly 4 s to sort the list $L$ using vm_compute. The downside of these definitions is made apparent as soon as we start to prove that our four functions lift, subst, hnf and nf enjoy a simple correctness property: that they always return terms that are well-formed and preserve well-formedness of the global state. Proving this requires around 600 lines of code, which brings the total size of this formalization to 900 lines, before we even started to prove meta-properties like the fact that hnf or nf implement beta-reduction! 


\subsection{Discussion}

In this section, we have demonstrated that our design patterns can be applied to other settings than BDDs. This exercise helped us to refine the presentation of smart constructors we gave in $\$ 6.1$ and gave us the opportunity to present another flavor of the shallow-embedding technique, that does not rely on the mutually recursive inductives we used in $\$ 5.2$

In short, this is a nice exercice de style. Yet, we believe that this study is also significant by itself. Reduction in the $\lambda$-calculus is representative of the kind of computation that arises in Coq, e.g. in the conversion test. Our case study can be seen as a step stone for future works that would attempt to prove the correctness of efficient implementations of symbolic algorithms. On the one hand, one could use the PURE-SHALLOW approach to implement hash-consing and memoization, yet remain inside the bounds of what Coq's extraction mechanism can safely handle. On the other hand, one could bite the bullet, use the SMART approach, and focus on more interesting details than hash-consing and memoization.

\section{Conclusion}

In this paper, we proposed two solutions to implement hash-consing in programs certified with the Coq system. The first one is to implement it using Coq data structures; the second is to use the imperative features provided by OCaml through the tuning of the extraction mechanism. The difference in flavor between the mapping of Coq constants to smart OCaml realizers or the axiomatization of there realizers in Coq is a matter of taste. In both cases, some meta-theoretical reasoning is required and requires to "sweep something under the rug".

\subsection{Trusting manually-tweaked extracted code}

Wrapping-up this article, we have to stress the fact that using hand-written code in the extraction process yields code that is less trustworthy. Indeed, the code we use to realize axioms (e.g., inhabitants of the type memoizer) or to replace constructors and destructors with the suitable smart ones may introduce bugs.

For instance, we could end up producing code that fails to compile, because the code snippets used to customize the extraction are used as textual replacements. Or, we could end up producing code that is observationally not-equivalent to the pristine extracted function because of side-effects. Or we could simply ruin all our verification effort by introducing the same kind of bugs that we wanted to catch using formal proofs.

There are two simple ways to restore confidence in this code: code reviews and tests. Unfortunately, doing more than that is hard. For instance, we could imagine using the Why3 20] framework to reason about our code. Unfortunately, Why3 is not suited for reasoning about higher-order programs, such as our memoizing fixpoint operators. More promising is Charguéraud's CFML 7 framework, that makes it possible to reason about ML programs with effects. We wonder to what extent the verification of our combinators could be conducted in this system. 
In any case, we would like to point out that using hand-written code only increase the trusted code base, which contains the whole extraction mechanism.

\subsection{Trusting external imperative code}

The simple act of calling external libraries that rely on side effects may introduce inconsistencies if these libraries return inconsistent values when called with identical parameters. This is because, in Coq, a given term has a unique value inside a model (or a trace of execution, in practical terms). This is the reason why exposing the internals of mutable state, such as unique identifiers, is fraught with possibilities of introducing subtle inconsistencies.

For example, the "else" branch of the following Coq program is unreachable, because foo $\mathrm{x}$ and foo (pred $(\mathrm{S} \mathrm{x})$ ) are equal, thus the theorem stating that it always returns "true":

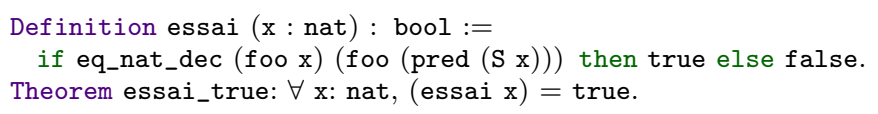

Yet, if foo is extracted to a function such as

let counter $=$ ref 0 in fun $x \rightarrow$ counter $:=\mathrm{S}($ !counter $) ;$ counter

then the OCaml code for essai 0 returns "false".

\subsection{Some practical details of implementation}

We took care through this article not to use Coq's advanced support for recursive definitions, i.e., the Program and Function commands. There are two important reasons for this. First, as we hinted at in $\$ 5.1$. the termination arguments for our BDD operations are out of the scope of what both commands can automate. Second, we could have used Program instead of refine in many of our definitions. Both can be used to build terms with holes, that are later filled using tactics. However, Program is based on a powerful elaboration [19], meaning that the final term that is built may be quite different from the one the user has written.

Generally speaking, the performances of the OCaml version of the libraries we build using the SMART approach are quite dependent on side-effects. For instance, keeping memoization tables from one run of a given BDD operation to the other yields different performance and memory consumption profiles from what one gets without this conservative strategy. The only change between these conservative and non-conservative strategies is when the memoization tables are allocated. Therefore, we are quite picky when it comes to program transformations that could introduce partial applications or eta-expansions. Using refine, we are more confident that the code that we wrote is the one that is going to be extracted, and we have a better idea of the kind of OCaml code that is produced. This makes it easier to deal with this kind of potential performance issues. 


\subsection{Related work}

Purely functional implementations of BDDs Christiansen and Huch 8, Jeremy Bradley [15] implemented lazy BDDs in Haskell in a manner similar to our "pureshallow" approach. In their approaches, not only are BDDs maximally shared, they are also lazily evaluated "on demand". We did not investigate this possibility.

Imperative features inside the specification language. An obvious solution to implement and reason about imperative algorithms is to have these imperative features present in the modeling language of the prover. Some provers directly target highlevel programming languages with data structures, references and imperatives features: an example is KeY [2], which targets a large subset of Java. While such features are not available in Coq, there are two conceptual difficulties with this approach that would have made it impractical in our case-studies. First, BDD algorithms implemented using hash-consing are functional in a high-level view: BDD operations are very clearly given functionally, by induction; but also because hash-consing is suitable only for immutable structures ${ }^{13}$. It therefore seems strange to have to program them in an imperative language, furthermore one that complicates common functional idioms (e.g. pattern-matching). Second, the meta-theory for such languages is typically huge, with intricate proof rules having to deal with mutable data, references, late binding etc. It is not obvious how much we can trust the proof system with respect to the semantics of the language.

A second option is to add to an existing functional specification language certain imperative traits as monads, then modify the extraction function from the specification language to the target language so as to translate monadic operations into imperative calls, as has been done for Isabelle/HOL 6]; this approach has been used to verify a BDD package [10]. Special proof idioms have to be used for monadic programs, in addition to the general difficulty of programming in monadic style (see $\$ 2.1$ ). To the best of our knowledge, this approach has not been investigated in Coq.

A third option is to integrate into the specification language some essentially imperative data structures (e.g. mutable arrays, from which hash tables can be implemented), but present them in a functional fashion (e.g. an update to a mutable array is treated as returning a new array, same as the previous except for the updated location). The imperative features are then implemented efficiently for evaluation of expressions inside the prover, and are mapped to native imperative features of the target language during extraction. For instance, an experimental version of Coq exists, with native integers and arrays 1]. Again, all difficulties with monads (see $\$ 2.1$ apply, plus there is the problem of running a nonstandard version of Coq.

A fourth option is to deeply embed a subset of an imperative language into Coq: the programs of this imperative language are given a semantics inside Coq, and correctness properties are proved with respect to this semantics. This idea is discussed by Vafeiadis [21], but, as he remarks, this largely precludes the use of regular proof tactics: we have to develop proof steps specific to the language being embedded, and prove these steps correct with respect to the semantics.

\footnotetext{
13 Or at least for structures behaving as though they were immutable; for instance, we can perform hash-consing on a structure if the mutable information in the structure is just used for caching and does not affect the hashcode.
} 
Verification of BDD algorithms in Coq. Verma et al. 22, 23 implemented and proved correct in Coq a BDD library featuring efficient negation and disjunction; other operations like conjunction, implication and so on are implemented as derived operations. The BDDs produced are reduced and shared.

As we said, we chose to implement a fresh one because the code associated to their paper did not age well w.r.t. the evolution of Coq. Beside the fact that they investigated garbage collection, there is no conceptual difference between their library and our PURE-DEEP approach.

Verification of $B D D$ algorithms in other theorem provers In Isabelle/HOL, Ortner and Schirmer [18 verified the implementation of a normalization algorithm for binary decision diagrams. That is, their algorithm takes as input a BDD, and outputs the corresponding ROBDD. Their formalization is built on the BurstallBornat memory model: they build one heap of type ref $\rightsquigarrow$ value for each component of a BDD node, with ref the abstract type of memory addresses. Using a split-heap model makes it easier to reason about heap-allocated data structures in tools such as Why3. We believe however, that our formalization is more direct than theirs, and more suitable for efficient implementations in Coq.

Boyer and Hunt [4] developed an extension of ACL2 that uses hash-consing to give canonical representatives to ACL2 objects. This makes it possible to memoize some ACL2 user defined functions. As a case study, they implemented a BDD library in ACL2. Remark that this implementation is based on the fact that ACL2 exposes hash-consing primitives and the associated reasoning principles to the user. It is unclear to what extent these features could be added to the Coq proof assistant.

Hash-consing in the execution language Jean Goubault-Larrecq's HimML programming language $[11-13]$ is an extension of core Standard ML with primitive finite set and map data types with a run-time system designed around the concept of maximal sharing, or systematic hash-consing.

\subsection{Future work}

We conclude with directions for future work. First, we believe that the smart constructors approach is generalizable to a huge variety of inductive types. One can imagine it could be the job of Coq's extraction mechanism to implement on-demand such smart constructors and memoizers as it was the case for other imperative constructs [1]. Where to stop? Should the extraction mechanism also provide built-in functional maps and sets upon hash-consed types?

Second, in $\$ 5.2$, we hinted at the fact that using two mutually inductive datatypes for expressions and hash-consed expressions yields more complicated proofs than the one we had to do in $\S 8.3$. However, these two mutually inductive data-types are at the heart of Conchon and Filliâtre hash-consing library. We believe that following their approach closely is a good starting point for investigating to what extent one could provide a certified version of this hash-consing library.

Third, we confess that our implementations of BDDs are a bit rough. We would like to polish the implementations of our PURE-SHALLOW and SMART approaches, 
and release them as user-friendly libraries. In particular, we need to add some operations like quantification or functional composition.

\section{References}

[1] Michaël Armand et al. "Extending Coq with Imperative Features and Its Application to SAT Verification". In: Proc. ITP. Vol. 6172. LNCS. Springer, 2010, pp. 83-98. DOI: 10.1007/978-3-642-14052-5_8.

[2] Bernhard Beckert, Reiner Hähnle, and Peter H. Schmitt, eds. Verification of Object-Oriented Software: The KeY Approach. Vol. 4334. LNCS. Springer, 2007. DOI: 10.1007/978-3-540-69061-0.

[3] Bruno Blanchet et al. "Design and Implementation of a Special-Purpose Static Program Analyzer for Safety-Critical Real-Time Embedded Software". In: The Essence of Computation: Complexity, Analysis, Transformation. LNCS 2566. Springer, 2002, pp. 85-108.

[4] Robert S. Boyer and Warren A. Hunt Jr. "Function memoization and unique object representation for ACL2 functions". In: ACL2. ACM, 2006, pp. 81-89.

[5] Thomas Braibant, Jacques-Henri Jourdan, and David Monniaux. Supplementary material. URL: https://github.com/braibant/hash-consing-coq.

[6] Lukas Bulwahn et al. "Imperative Functional Programming with Isabelle/HOL". In: Theorem Proving in Higher Order Logics (TPHOL). Vol. 5170. LNCS. Springer, 2008, pp. 134-149. DOI: 10.1007/978-3-540-71067-7_14.

[7] Arthur Charguéraud. "Characteristic Formulae for the Verification of Imperative Programs". In: Proceeding of the 16th ACM SIGPLAN International Conference on Functional programming (ICFP). Ed. by Manuel M. T. Chakravarty, Zhenjiang $\mathrm{Hu}$, and Olivier Danvy. ACM, 2011, pp. 418-430.

[8] Jan Christiansen and Frank Huch. "A purely functional implementation of ROBDDs in Haskell". In: Trends in Functional Programming. Ed. by Henrik Nilsson. Vol. 7. Trends in Functional Programming. Intellect, 2006, pp. 55-71. ISBN: 978-1-84150-188-8.

[9] Sylvain Conchon and Jean-Christophe Filliâtre. "Type-Safe Modular HashConsing". In: ACM SIGPLAN Workshop on ML. Portland, Oregon, Sept. 2006. URL: http://www.lri.fr/ filliatr/ftp/publis/hash-consing2.pdf.

[10] Mathieu Giorgino and Martin Strecker. "Correctness of Pointer Manipulating Algorithms Illustrated by a Verified BDD Construction". In: Formal Methods $(F M)$. Ed. by Dimitra Giannakopoulou and Dominique Méry. Vol. 7436. LNCS. Springer, 2012, pp. 202-216. DOI: 10.1007/978-3-642-32759-9_18.

[11] Jean Goubault. "HimML: Standard ML with Fast Sets and Maps". In: 5th ACM SIGPLAN Workshop on ML and its Applications. 1994. URL: http: //citeseerx.ist.psu.edu/viewdoc/summary?doi=10.1.1.40.4967.

[12] Jean Goubault. Implementing Functional Languages with Fast Equality, Sets and Maps: an Exercise in Hash Consing. Tech. rep. Bull S.A. Research Center, May 1994. URL: http://citeseerx.ist.psu.edu/viewdoc/summary?doi=10.1 1.41.1757.

[13] Jean Goubault-Larrecq. HimML: HimML is a map-oriented $\boldsymbol{M L}$. Dec. 2008. URL: http://www.lsv.ens-cachan.fr/ goubault/himml-dwnld.html.

[14] Gérard Huet. "Constructive Computation Theory". Course notes, available at http://yquem.inria.fr/ huet/PUBLIC/CCT.pdf. 
[15] Neil Davies Jeremy Bradley. Compositional BDD Construction: A Lazy Algorithm. Tech. rep. CSTR-98-005. Department of Computer Science, University of Bristol, 1998. URL: http://www.cs.bris.ac.uk/Publications/Papers/ 1000250.pdf.

[16] Donald E. Knuth. "The Art of Computer Programming". In: vol. 4A. Binary decision diagrams. Addison-Wesley, 2011. Chap. 7.1.4.

[17] Guillaume Melquiond. "Floating-point arithmetic in the Coq system". In: Information and Computation 216 (2012), pp. 14-23. DOI: 10.1016/j.ic.2011 09.005 .

[18] Veronika Ortner and Norbert Schirmer. "Verification of BDD Normalization". In: TPHOLs. Vol. 3603. Lecture Notes in Computer Science. Springer, 2005, pp. 261-277.

[19] Matthieu Sozeau. "Subset Coercions in Coq". In: TYPES. Vol. 4502. Lecture Notes in Computer Science. Springer, 2007, pp. 237-252.

[20] The Why3 platform 0.80. URL: http://why3.lri.fr

[21] Viktor Vafeiadis. "Adjustable references". In: ITP. Vol. 7998. Lecture Notes in Computer Science. Springer, 2013, pp. 328-337.

[22] Kumar Neeraj Verma and Jean Goubault-Larrecq. Reflecting BDDs in Coq. Rapport de recherche RR-3859. INRIA, 2000. HAL: inria-00072797.

[23] Kumar Neeraj Verma et al. "Reflecting BDDs in Coq". In: Proc. ASIAN. Vol. 1961. LNCS. Springer, 2000, pp. 162-181. DOI: 10.1007/3-540-444645_13. 